\title{
D2 Receptor Block Abolishes Theta Burst Stimulation-Induced Neuroplasticity in the Human Motor Cortex
}

\author{
Katia Monte-Silva', ${ }^{1,3}$, Diane Ruge ${ }^{2}$, James T Teo ${ }^{2}$, Walter Paulus', John C Rothwell ${ }^{2}$ and Michael A Nitsche*, \\ 'Department of Clinical Neurophysiology, Georg-August University, Göttingen, Germany; ${ }^{2}$ Sobell Department of Motor Neuroscience and \\ Movement Disorders, Institute of Neurology, University College London, London, UK; ${ }^{3}$ Department of Physical Therapy, Federal University of \\ Pernambuco, Recife, Brazil
}

\begin{abstract}
Dopamine (DA) is a neurotransmitter with an important influence on learning and memory, which is thought to be due to its modulatory effect on plasticity at central synapses, which in turn depends on activation of DI and D2 receptors. Methods of brain stimulation (transcranial direct current stimulation, tDCS; paired associative stimulation, PAS) lead to after-effects on cortical excitability that are thought to resemble long-term potentization (LTP)/long-term depression (LTD) in reduced preparations. In a previous study we found that block of D2 receptors abolished plasticity induced by tDCS but had no effect on the facilitatory plasticity induced by PAS. We postulated that the different effect of D2 receptor block on tDCS- and PAS-induced plasticity may be due to the different focality and associativity of the stimulation techniques. However, alternative explanations for this difference could not be ruled out. tDCS also differs from PAS in other aspects, as tDCS induces plasticity by subthreshold neuronal activation, modulating spontaneous activity, whereas PAS induces plasticity via phasic suprathreshold stimulation. The present study in 12 volunteers examined effects of D2 receptor blockade (sulpiride (SULP) $400 \mathrm{mg}$ ), on the LTP/LTD-like effects of theta burst transcranial magnetic stimulation (TBS), which has less restricted effects on cortical synapses than that of PAS, and does not induce associative plasticity, similar to tDCS, but on the other hand induces cortical excitability shifts by suprathreshold (rhythmic) activation of cortical neurons similarly to PAS. Administration of SULP blocked both the excitatory and inhibitory effects of intermittent (iTBS) and continuous TBS (CTBS), respectively. As the reduced response to TBS following SULP resembles its effect on $\mathrm{DDCS}$, the results support an effect of DA on plasticity, which might be related to the focality and associativity of the plasticity induced.

Neuropsychopharmacology (20II) 36, 2097-2102; doi:I0.1038/npp.201 I.100; published online 22 June 201 I
\end{abstract}

Keywords: dopamine receptor; sulpiride; plasticity; motor cortex; transcranial magnetic stimulation; theta burst stimulation

\section{INTRODUCTION}

Dopamine (DA) is a neurotransmitter with an important influence on learning and memory, which is thought to be due to its ability to modulate plasticity at central synapses. Its actions at many synapses depend on whether it activates the D1 or D2 receptor (for review, see Iversen and Iversen, 2007). In the present study we focus on its effects via the D2 receptor.

Animal and slice experiments have demonstrated heterogeneous, sometimes even opposing effects of D2 receptor activation on neuroplasticity, that is, long-term potentiation (LTP) and depression (LTD) (Frey et al, 1989; Chen et al, 1996; Otani et al, 1998; Gurden et al, 2000; Spencer and

\footnotetext{
*Correspondence: Dr MA Nitsche, Department of Clinical Neurophysiology, Georg-August University, Robert-Koch-Strasse 40, 37075 Göttingen, Germany, Tel: + 4955 139 957I, Fax: + 4955 I39 8621, E-mail: mnitsch I@gwdg.de

Received 8 February 2011; revised 20 April 2011; accepted 3 May 2011
}

Murphy, 2000; Manahan-Vaughan and Kulla, 2003). Similarly, previous work in humans has shown that D2 receptors have a complex influence on the LTP/LTD-like plasticity induced by transcranial direct current stimulation (tDCS) (Kuo et al, 2008; Monte-Silva et al, 2009; Nitsche et al, 2006, 2009), and paired associative stimulation (PAS) (Stefan et al, 2000; Wolters et al, 2003). Transcranial direct current stimulation is applied in humans to elicit non-focal neuroplastic cortical excitability shifts. Here, anodal stimulation enhances excitability in the primary motor cortex, while cathodal stimulation reduces it (Nitsche and Paulus, 2000, 2001; Nitsche et al, 2003). The excitatory and inhibitory after-effects induced by anodal and cathodal tDCS, respectively, were shown to be abolished by blocking D2 receptor activity with sulpiride (SULP) (Nitsche et al, 2006). This strengthens the evidence for an impact of D2 receptor on non-focal plasticity. These results are in principal accordance with animal studies demonstrating that LTP and LTD are enhanced by D2 receptor activation (Otani et al, 1998; Spencer and Murphy, 2000; ManahanVaughan and Kulla, 2003). 
Modulation of D2 receptor function also interacts with LTP/LTD-like effects of a second plasticity-probing protocol, PAS. It produces focal plasticity in the sensorimotor cortex by combining activation of sensory afferents from peripheral nerve stimulation with activation of motor cortex neurons by transcraninal magnetic stimulation (TMS) (Stefan et al, 2000). PAS shares some features with spike-timing-dependent synaptic plasticity. When the sensory input elicited by peripheral nerve stimulation reaches the motor cortex simultaneously with TMS delivered directly to the motor cortex, it elicits a long-lasting cortical excitability enhancement (facilitatory PAS; Stefan et al, 2000). However, if the afferent stimulus reaches the respective motor cortical neurons relevantly later than the TMS stimulus, it induces long-lasting cortical inhibition (inhibitory PAS; Wolters et $\mathrm{al}, 2003)$. In contrast to tDCS studies, blocking D2 receptors with SULP slightly enhanced the facilitatory effects of synchronous PAS in humans, but abolished the excitability reduction induced by asynchronous PAS (Nitsche et al, 2009), being in favor of a minor impact of D2 receptors on facilitatory PAS-induced plasticity. However, as enhancement of D2 receptor activity had prominent non-linear effects of PAS-induced plasticity in another study, this does not rule out an influence of D2 receptors on this kind of plasticity completely (Monte-Silva et al, 2009). In contrast, a recent study conducted in animal slice preparations suggests that $\mathrm{D} 2$ receptor activation has a role for induction of focal spike-timing-dependent plasticity (STDP) (Xu and Yao, 2010). These non-uniform effects in animal slice preparations and humans point out the complex action of D2 receptor on cortical plasticity and support the necessity of more studies to help understanding the impact of dopaminergic sub-receptors on synaptic plasticity.

We have hypothesized that the different effect of D2 receptor block on tDCS- and PAS-induced plasticity may be caused by the different spatial and temporal focality of the stimulation techniques (Nitsche et al, 2009). Although tDCS induces plasticity by a tonic stimulation procedure, lasting for some minutes, which is not restricted to specific synaptic subgroups, PAS-induced plasticity is accomplished by phasic stimulation, which is the repetitive suprathreshold activation of neurons by short-lasting stimuli, and is thought to be restricted to certain synaptic subgroups, which are affected by both, the peripheral nerve, and the TMS stimuli. However, alternative explanations for the different effects of D2 block on the plasticity induced by both techniques cannot be ruled out; tDCS induces subthreshold shifts of membrane polarity, which in turn alter cortical excitability and spontaneous activity (Bindman et al, 1964; Nitsche and Paulus, 2000, 2001; Nitsche et al, 2003). In contrast, PAS activates somatosensorymotor cortical connections by suprathreshold induction of action potentials (Stefan et al, 2000).

In order to test the origin of the differences of the effects of D2 receptor block on both kinds of plasticity in more detail, in the present experiment we have explored the effect of D2 receptor block on another transcranial plasticity induction protocol, theta burst transcranial magnetic stimulation (TBS) (Huang et al, 2005). It was derived from classical protocols for induction of synaptic plasticity in brain slice preparations and leads to LTP/LTD-like effects on cortical plasticity (Huang et al, 2007; Teo et al, 2007).
It is known that TBS, as well as tDCS and PAS, induces longlasting, $N$-methyl-D-aspartic acid (NMDA) receptor-dependent neuroplastic excitability shifts (Stefan et al, 2002; Nitsche et al, 2003; Huang et al, 2007). The difference between the respective plasticity-inducing stimulation protocols is at least threefold; (1) tDCS- and TBS-elicited neuroplasticity is synaptically driven but not restricted to specific subgroups of synapses as opposed to the plasticity induced by PAS; (2) plasticity induced by PAS is associative and time-dependent, while the tonic neuronal polarization by tDCS, and the high-frequency phasic stimulation of TBS, should not induce associative plasticity (Bindman et al, 1964; Weise et al, 2006); (3) tDCS, in contrast to TBS, and PAS, does not induce cortical activity by suprathreshold activation of cortical neurons, but modulates spontaneous cortical activity.

Thus, we hypothesized that if focality and associativity of stimulation are the main factors that explain heterogenous effects of DA on stimulation-induced plasticity, then TBS should be affected in the same way as tDCS by D2 receptor block. In contrast, if phasic suprathrehold $v s$ tonic subthreshold stimulation are the relevant parameters, we would expect a similar effect of D2 block on TBS-generated plasticity as accomplished for PAS.

\section{SUBJECTS AND METHODS}

\section{Subjects}

Twelve healthy volunteers participated in the experiment (six men, aged $25.75 \pm 5.11$ years). They received iTBS and cTBS combined with either $400 \mathrm{mg}$ SULP or placebo (PLC) medications in different sessions of the study (four sessions per subject). The study was conducted in a complete crossover design (for more details see Experimental Design). None were taking any acute or regular medication at the time of the study, or had a history of chronic or acute neurological, psychiatric, or medical diseases, family history of epilepsy, pregnancy, cardiac pacemaker, or metallic implants. All participants gave their written informed consent before the commencement of the experiment. The experiments were performed with the approval of the Joint Research Ethics Committee of the National Hospital for Neurology and Neurosurgery and the Institute of Neurology of the London University College and were performed in accordance with the Declaration of Helsinki.

\section{Pharmacological Intervention}

A total of $400 \mathrm{mg}$ SULP or equivalent PLC drugs were ingested by the subjects $1.5 \mathrm{~h}$ before TBS so that the latter coincided with the peak plasma concentration of SULP (Deleu et al, 2002). This is the same dose as in previous studies in which there had been clear effects on the response to tDCS and PAS (Nitsche et al, 2006, 2009). The experimental sessions were separated by at least 1 week to avoid cumulative drug or stimulation effects.

\section{Theta Burst Stimulation}

TBS was applied using a Magstim Rapid2 Package (Magstim, Whitland, UK) and consisted of bursts 


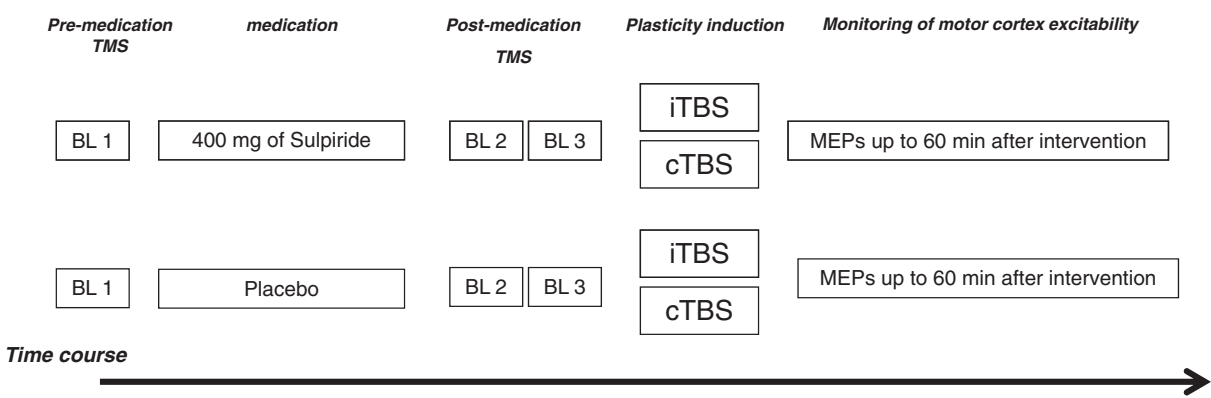

Figure I Experimental course of the present study. Transcraninal magnetic stimulation (TMS) was applied over the left motor cortical representational area of the right first dorsal interosseous muscle (FDI) with an intensity to elicit motor-evoked potentials (MEPs) with a peak-to-peak amplitude of on average I $\mathrm{mV}$ (baseline - BL I). At I.5 h after intake of $400 \mathrm{mg}$ SULP or placebo (PLC) medication, a second baseline (baseline 2-BL 2) was determined to control for a possible influence of the drug on cortical excitability, and adjusted if necessary (baseline 3-BL 3). Two theta burst transcranial magnetic stimulation (TBS) protocols were used: (I) intermittent TBS (iTBS) and (2) continuous TBS (CTBS). Immediately after TBS, MEPs were recorded for 30 min at every $5 \mathrm{~min}$, then at every $15 \mathrm{~min}$ for up to $60 \mathrm{~min}$ after the end of stimulation.

containing three pulses at $50 \mathrm{~Hz}$ frequency with an intensity of $80 \%$ active motor threshold (AMT, see Monitoring of motor cortical excitability) repeated at $200 \mathrm{~ms}$ intervals. Two TBS protocols were used:

1. Intermittent TBS (iTBS): a 2-s train of TBS repeated every $10 \mathrm{~s}$ for a total of $190 \mathrm{~s}$ (600 pulses)

2. Continuous theta burst stimulation (cTBS): a 40-s train of uninterrupted TBS (600 pulses).

These patterns of TBS induce opposite effects on motor cortex excitability. iTBS generates a facilitatory effect on the motor-evoked-potential (MEP) amplitudes induced by TMS, whereas cTBS decreases MEP amplitudes for 20-30 min (Huang et al, 2005).

\section{Monitoring of Motor Cortical Excitability}

TMS-elicited MEPs were recorded to measure excitability changes of the motor cortex representation of the right first dorsal interosseous muscle (FDI). Single-pulse TMS was applied with a Magstim 200 magnetic stimulator (Magstim, UK) using a figure-of-eight magnetic coil (diameter of one winding $=70 \mathrm{~mm}$, peak magnetic field $=2.2 \mathrm{~T})$. The coil was held tangentially to the skull, with the handle pointing backward and laterally at an angle of $45^{\circ}$ from midline. The optimal coil position was determined by the location on the scalp where magnetic stimulation resulted consistently in the largest MEP when the subject was relaxed ('motor hotspot'). Electromyographic (EMG) recording was made from the right FDI with Ag-AgCl electrodes in a belly-tendon montage. Responses were amplified with a Digitimer D360 amplifier (Digitimer, Welwyn Garden City, UK) and filtered with set at $20 \mathrm{~Hz}$ and $2 \mathrm{kHz}$ with a sampling rate of $5 \mathrm{kHz}$, then recorded by a computer using SIGNAL software (Cambridge Electronic Devices, Cambridge, UK). The intensity was adjusted to elicit, on average, baseline MEPs of 1-mV peak-to-peak amplitude, and was kept constant for the post-stimulation assessment, unless adjusted (see Experimental Design).

\section{Experimental Design}

The experiment was conducted in a double-blinded, PLC-controlled complete crossover design. Each subject participated in four experimental sessions separated by at least 1 week from each other. In each session, the subjects received iTBS or cTBS and either $400 \mathrm{mg}$ SULP or PLC drugs (PLC/iTBS; PLC/cTBS; SULP/iTBS and SULP/ cTBS). The order of the four experimental sessions was randomized between participants. Medication and TBS were administrated by a scientist who neither performed the measurement of motor cortical excitability nor took part in the data analysis. Thus, the investigator was blinded with regard to the specific medication and TBS paradigm.

Subjects were seated in a comfortable chair with head and arm rests. After the motor cortex representation area of the right FDI was identified, the intensity of TMS was adjusted to elicit 20 MEPs with a peak-to-peak amplitude of on average $1 \mathrm{mV}$ (baseline 1). One and a half hour after intake of SULP or the equivalent PLC medication, a second baseline (baseline 2) was determined to control for a possible influence of the drug on cortical excitability. If the baseline 2 MEP amplitude was altered by SULP, we adjusted TMS intensity to obtain a baseline 3 MEP amplitude of about $1 \mathrm{mV}$. This TMS intensity was kept constant for the post-stimulation assessment.

Before start of TBS (either excitatory or inhibitory), the AMT was determined. AMT was defined as the minimum single-pulse TMS intensity required to produce an MEP amplitude larger than $200 \mu \mathrm{V}$ in more than 5 out of 10 trials from the contralateral FDI while the subject was maintaining a voluntary contraction of about $20 \%$ of maximum. Afterward motor cortical TBS was performed. Immediately after TMS, 25 MEPs were recorded every $5 \mathrm{~min}$ at $0.25 \mathrm{~Hz}$ for half an hour, and then at every $15 \mathrm{~min}$ for up to $60 \mathrm{~min}$ after the end of stimulation (Figure 1). Coil position was marked with a waterproof pen to guarantee identical position during the whole course of the experiment.

\section{Data Analysis and Statistics}

Individual MEP amplitude means were calculated for each time bin, including baseline 1, 2, and 3 and poststimulation time points, separately for each stimulation/ medication combination. The post-intervention MEPs were normalized to the pre-TBS measurements. They are given as ratios of the pre-TBS baseline (baseline 3 or 2, when not adjusted). A repeated measures analysis of variance 


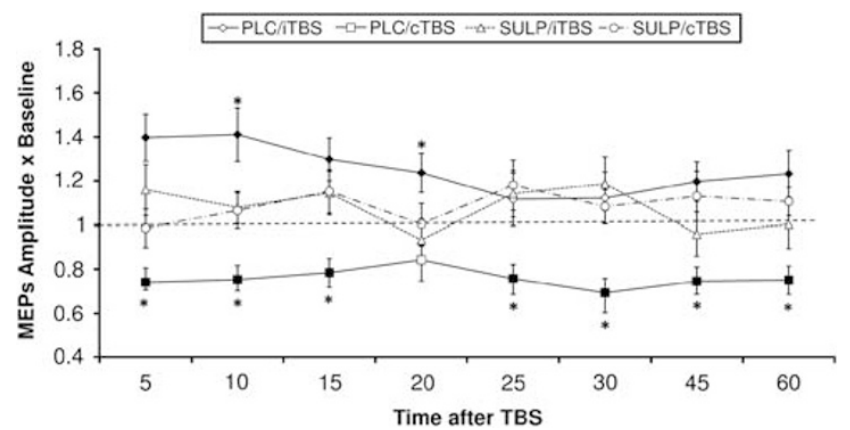

Figure 2 Impact of D2 block on plasticity induced by intermittent theta burst transcranial magnetic stimulation (iTBS) and continuous (CTBS). The time-course plots show the effect of $400 \mathrm{mg}$ sulpiride (SULP) on TBSinduced neuroplasticity. Depicted are the baseline-standardized motorevoked potential (MEP) amplitudes after plasticity induction by iTBS or CTBS under SULP or placebo (PLC) medication for up $60 \mathrm{~min}$ after the end of TBS. As shown by transcraninal magnetic stimulation (TMS)-elicited MEP amplitudes, under PLC medication, iTBS enhances, whereas CTBS diminishes cortical excitability significantly for up to $60 \mathrm{~min}$ after stimulation. Under SULP, the TBS-generated excitability shifts are abolished. Filled symbols indicate significant deviations of the post-TBS MEP amplitudes from baseline, asterisks indicate significant deviations of SULP vS PLC condition with regard to identical time points and TBS protocols (t-test, two-tailed, paired samples, $p<0.05)$. The error bars show SEM.

(ANOVA) was calculated using the within-subject factors 'time course' (up to 60 after TBS), 'TBS protocol' (iTBS and CTBS), 'drug condition' (400 mg of SULP and PLC), and the dependent variable MEP amplitude. Post-hoc Student's $t$-tests (paired samples, two-tailed, $p<0.05$, not corrected for multiple comparisons) were performed to test for baseline differences between the medication/TBS conditions, for an impact of SULP on cortical excitability independent from TBS, to compare the MEP amplitudes before and after TBS within each protocol, and for each time bin to identify MEP differences between protocols. A $p$-value of $<0.05$ was considered significant for all statistical analyses.

\section{RESULTS}

The ANOVA revealed significant main effects of 'TBS protocol' $(\mathrm{df}=1, \mathrm{~F}=10.339, p=0.008)$ and significant interactions of 'time course' $\times$ 'drug condition' $(\mathrm{df}=8$, $\mathrm{F}=2.076, p=0.047)$, 'time course' $\times$ 'TBS protocol' $(\mathrm{df}=8$, $\mathrm{F}=2.579, \quad p=0.014), \quad$ 'drug condition' $\times$ 'TBS protocol' $(\mathrm{df}=1, \quad \mathrm{~F}=14.717, \quad p=0.003)$, and 'time course' $\times$ 'TBS protocol' $\times$ 'drug condition' $(\mathrm{df}=8, \mathrm{~F}=2.781, p=0.009)$. Confirming previous studies (Huang et al, 2007; Teo et al, 2007), iTBS with PLC medication significantly enhanced motor cortex excitability, whereas cTBS reduced it for up to $1 \mathrm{~h}$ after the end of stimulation. Conversely, after $400 \mathrm{mg}$ of SULP, the excitatory and inhibitory effects of TBS on cortical excitability were suppressed and differed significantly from the PLC medication condition (Figure 2). Peak-to-peak baseline MEP amplitudes were identical in all conditions $(p \geqslant 0.05$, Student's $t$-tests, paired, two-tailed). Sulpiride alone did not modify corticospinal excitability significantly (comparison of baseline a before, and 2 after SULP ingestion), and
Table I The Peak-to-Peak MEP Amplitudes Before and After Application of Sulpiride (SULP)

\begin{tabular}{ccccc}
\hline & $\begin{array}{c}\text { Baseline I } \\
(\mathbf{m V})\end{array}$ & $\begin{array}{c}\text { Baseline } 2 \\
(\mathbf{m V})\end{array}$ & $\begin{array}{c}\text { Baseline 3 } \\
(\mathbf{m V})\end{array}$ & MSO\% \\
\hline $\begin{array}{cccc}\text { Placebo } \\
\text { iTBS }\end{array}$ & $1.03 \pm 0.10$ & $1.05 \pm 0.10$ & $1.07 \pm 0.06$ & $46 \pm 2.4$ \\
CTBS & $1.01 \pm 0.08$ & $0.95 \pm 0.08$ & $1.00 \pm 0.07$ & $47 \pm 2.2$ \\
& & & & \\
400 mg of SULP & & & \\
TTBS & $1.14 \pm 0.10$ & $0.92 \pm 0.07$ & $1.10 \pm 0.10$ & $46 \pm 2.0$ \\
CTBS & $0.98 \pm 0.11$ & $0.95 \pm 0.08$ & $0.95 \pm 0.05$ & $45 \pm 1.6$ \\
\hline
\end{tabular}

Shown are the mean MEP amplitudes \pm SEM of baselines I, 2, and 3, and the mean \pm SEM of the intensities of the magnetic cortical stimulus as percentage of maximum stimulator output (MSO). The intensity of TMS was adjusted to elicit MEPs with a peak-to-peak amplitude of on average I $\mathrm{mV}$ (baseline I). At $1.5 \mathrm{~h}$ after intake of SULP ( $400 \mathrm{mg}$ ), a second baseline (baseline 2) was determined to control for an influence of the drug on cortical excitability, and adjusted if necessary (baseline 3). MEP amplitudes and percentage of MSO did not differ significantly between TBS conditions as well as before and after drug intake within the specific TBS/drug combinations ( $p \geqslant 0.05$, Student's $t$-tests, paired, two-tailed)

baseline MEP amplitudes as well as TMS stimulation intensity did not differ between conditions (Table 1).

\section{DISCUSSION}

The results of this study demonstrate that blocking D2 receptors with SULP impairs the LTP- and LTD-like aftereffects of excitatory and inhibitory TBS protocols (iTBS and cTBS, respectively) in the human motor cortex. This is consistent with evidence from animal experiments showing that cortical LTP and LTD can be enhanced by D2 receptor activation (Otani et al, 1998; Spencer and Murphy, 2000; Manahan-Vaughan and Kulla, 2003; Xu and Yao, 2010). It complements the effects of D2 receptor blockade and stimulation on cognitive processes, which are impaired by both SULP and haloperidol (Kumari et al, 1997; Mehta et al, 1999). The reduced response to TBS is similar to the effect of SULP on tDCS-induced plasticity (Nitsche et al, 2006) but differs from those induced by facilitatory PAS, which are not abolished but slightly consolidated by D2 antagonism (Nitsche et al, 2009).

Our primary hypothesis was that block of D2 receptors has different effects depending on the focality of a plasticity induction protocol, with D2 block having a greater influence on non-focal than focal plasticity. This would be in line with the assumption that focal plasticity is primarily controlled by D1 receptors (Seamans and Yang, 2004) and is dependent on activity levels of neuronal networks (Surmeier and Kitai, 1997). However, alternative explanations for the different effects of D2 receptor block on plasticity cannot be ruled out definitely, because plasticity induction protocols like tDCS and PAS differ also in a number of other respects.

It is known that TBS, as well as tDCS and PAS, induce long-lasting, NMDA receptor-dependent neuroplastic excit- 
ability shifts (Stefan et al, 2002; Nitsche et al, 2003; Huang et al, 2007). The difference between these plasticityinducing stimulation protocols is at least threefold:

1. tDCS- and TBS-elicited neuroplasticity is synaptically driven but not restricted to specific subgroups of synapses, as opposed to the plasticity induced by PAS which is thought to be restricted to the subgroup of synapses targeted by timed afferent somatosensory input;

2. plasticity induced by PAS is associative and timedependent, while the tonic neuronal polarization by tDCS, and the high-frequency phasic stimulation of TBS, should not induce associative plasticity (Bindman et al, 1964; Weise et al, 2006);

3. tDCS, in contrast to TBS, and PAS, does not induce cortical activity by suprathreshold activation of cortical neurons, but modulates spontaneous cortical activity.

Interestingly, the results of the present study show that TBS-induced plasticity is affected by D2 receptor block similarly to tDCS-generated changes of excitability. Thus, it might be concluded that the focality and associativity of stimulation are the main relevant factors explaining the different effects of D2 receptor on stimulation-induced plasticity.

The mechanisms underlying the D2 receptor effect on TBS-induced plasticity in humans are far from being completely understood. However, TBS-induced plasticity depends on the glutamatergic system (Huang et al, 2007; Teo et al, 2007). It is known that $\mathrm{D} 2$ receptor activation reduces the activation of NMDA receptors on the one hand (Seamans and Yang, 2004). However, it is improbable that a strengthening of NMDA receptors by D2 receptor block abolished TBS-induced plasticity. On the other hand, D2 receptor activation also diminishes GABAergic inhibition, and thus SULP will enhance GABAergic tone (Seamans and Yang, 2004; Tseng and O'Donnell, 2004).

As enhanced GABAergic tone compromises glutamatergic plasticity, as shown by many studies conducted in animals and humans (Del Cerro et al, 1992; Seabrook et al, 1997; Ziemann et al, 2001), this mechanism, which however should be proven more directly in future studies, is an attractive candidate for the plasticity-abolishing features of SULP.

In conclusion, our study addresses issues that are essential for the understanding of the non-uniform and complex effects of DA on synaptic plasticity and cognitive function in humans. Here, we have studied the influence of D2 receptor activity and suggest that its effect on plasticity in the human motor cortex depends on the focality of neuroplastic excitability changes. From a clinical perspective, our results demonstrate the relevance of the dopaminergic system for cortical neuroplasticity and furthermore might help to understand the pathophysiology of neuropsychiatric diseases accompanied by DA malfunction, like schizophrenia, restless legs syndrome, Parkinson's disease, and depression. More studies, eg, experiments with L-DOPA and specific receptor agonists, as well as dose-dependent studies, are needed to enhance our knowledge about the impact of DA on TBS-induced neuroplasticity.

\section{ACKNOWLEDGEMENTS}

$\mathrm{K}$ Monte-Silva is supported by Coordenação de Aperfeiçoamento de Pessoal de Nível Superior (CAPES), Brazil. MAN is supported by a grant from the Deutsche Forschungsgemeinschaft (DFG grant NI 683/6-1). DR was supported by grants from the DMRF (Dystonia medical research foundation) and TSA (Tourette syndrome association).

\section{DISCLOSURE}

The authors declare no conflict of interest.

\section{REFERENCES}

Bindman LJ, Lippold OCJ, Redfearn JWT (1964). The action of brief polarizing currents on the cerebral cortex of the rat (1) during current flow and (2) in the production of long-lasting after-effects. J Physiol 172: 369-382.

Chen Z, Ito K, Fujii S, Miura M, Furuse H, Sasaki H et al (1996). Roles of dopamine receptors in long-term depression: enhancement via D1 receptors and inhibition via D2 receptors. Receptors Channels 4: 1-8.

Del Cerro S, Jung M, Lynch G (1992). Benzodiazepines block longterm potentiation in slices of hippocampus and piriform cortex. Neuroscience 49: 1-6.

Deleu D, Northway MG, Hanssens Y (2002). Clinical pharmacokinetic and pharmacodynamic properties of drugs used in the treatment of Parkinson's disease. J Pharmacokinet 4: 261-309.

Frey U, Hartmann S, Matthies H (1989). Domperidone, an inhibitor of the D2-receptor, blocks a late phase of an electrically induced long-term potentiation in the CA1-region in rats. Biomed Biochim Acta 48: 473-476.

Gurden H, Takita M, Jay TM (2000). Essential role of D1 but not D2 receptors in the NMDA receptor-dependent long-term potentiation at hippocampal-prefrontal cortex synapses in vivo. J Neurosci 20: RC106.

Huang YZ, Chen RS, Rothwell JC, Wen HY (2007). The after-effect of human theta burst stimulation is NMDA receptor dependent. Clin Neurophysiol 118: 1028-1032.

Huang YZ, Edwards MJ, Rounis E, Bhatia KP, Rothwell JC (2005). Theta burst stimulation of the human motor cortex. Neuron 45: 201-206.

Iversen SD, Iversen LL (2007). Dopamine: 50 years in perspective. Trends Neurosci 30: 188-193.

Kuo MF, Paulus W, Nitsche MA (2008). Boosting focally-induced brain plasticity by dopamine. Cereb Cortex 18: 648-651.

Kumari V, Corr PJ, Mulligan OF, Cotter PA, Checkley SA, Gray JA (1997). Effects of acute administration of d-amphetamine and haloperidol on procedural learning in man. Psychopharmacology (Berl) 129: 271-276.

Manahan-Vaughan D, Kulla A (2003). Regulation of depotentiation and long-term potentiation in the dentate gyrus of freely moving rats by dopamine D2-like receptors. Cereb Cortex 13: 123-135.

Mehta MA, Sahakian BJ, McKenna PJ, Robbins TW (1999). Systemic sulpiride in young adult volunteers simulates the profile of cognitive deficits in Parkinson's disease. Psychopharmacology 146: 162-174.

Monte-Silva K, Kuo M, Thirugnanasambandam N, Liebetanz D, Paulus W, Nitsche MA (2009). Dose-dependent inverted Ushaped effect of dopamine (D2-like) receptor activation on focal and non-focal plasticity in humans. J Neurosci 29: 6124-6131. 
Nitsche MA, Kuo MF, Grosch J, Bergner C, Monte-Silva K, Paulus W (2009). D1-receptor impact on neuroplasticity in humans. J Neurosci 29: 2648-2653.

Nitsche MA, Lampe C, Antal A, Liebetanz D, Lang N, Tergau F et al (2006). Dopaminergic modulation of long-lasting direct currentinduced cortical excitability changes in the human motor cortex. Eur J Neurosci 23: 1651-1657.

Nitsche MA, Nitsche MS, Klein CC, Tergau F, Rothwell JC, Paulus W (2003). Level of action of cathodal DC polarisation induced inhibition of the human motor cortex. Clin Neurophysiol 114: 600-604.

Nitsche MA, Paulus W (2000). Excitability changes induced in the human motor cortex by weak transcranial direct current stimulation. J Physiol 527: 633-639.

Nitsche MA, Paulus W (2001). Sustained excitability elevations induced by transcranial DC motor cortex stimulation in humans. Neurology 57: 1899-1901.

Otani S, Blond O, Desce JM, Crepel F (1998). Dopamine facilitates long-term depression of glutamatergic transmission in rat prefrontal cortex. Neuroscience 85: 669-676.

Seabrook GR, Easter A, Dawson GR, Bowery BJ (1997). Modulation of long-term potentiation in CA1 region of mouse hippocampal brain slices by GABAA receptor benzodiazepine site ligands. Neuropharmacology 36: 823-830.

Seamans JK, Yang CR (2004). The principal features and mechanisms of dopamine modulation in the prefrontal cortex. Prog Neurobiol 74: 1-58.

Spencer JP, Murphy KP (2000). Bi-directional changes in synaptic plasticity induced at corticostriatal synapses in vitro. Exp Brain Res 135: 497-503.
Stefan K, Kunesch E, Benecke R, Classen J (2002). Mechanisms of enhancement of human motor cortex excitability induced by interventional paired associative stimulation. J Physiol 543: 699-708.

Stefan K, Kunesch E, Cohen LG, Benecke R, Classen J (2000). Induction of plasticity in the human motor cortex by paired associative stimulation. Brain 123: 572-584.

Surmeier DJ, Kitai ST (1997). [State-dependent regulation of neuronal excitability by dopamine]. Nihon Shinkei Seishin Yakurigaku Zasshi 17: 105-110.

Teo JTH, Swayne OB, Rothwell JC (2007). Further evidence for NMDA-dependence of the after-effects of human theta burst stimulation. Clin Neurophysiol 118: 1649-1651.

Tseng KY, O’Donnell P (2004). Dopamine-glutamate interactions controlling prefrontal cortical pyramidal cell excitability involve multiple signaling mechanisms. J Neurosci 24: 5131-5139.

Weise D, Schramm A, Stefan K, Wolters A, Reiners K, Naumann M et al (2006). The two sides of associative plasticity in writer's cramp. Brain 129: 2709-2721.

Wolters A, Sandbrink F, Schlottmann A, Kunesch E, Stefan K, Cohen LG et al (2003). A temporally asymmetric Hebbian rule governing plasticity in the human motor cortex. $J$ Neurophysiol 89: 2339-2345.

$\mathrm{Xu}$ TX, Yao WD (2010). D1 and D2 dopamine receptors in separate circuits cooperate to drive associative long-term potentiation in the prefrontal cortex. Proc Natl Acad Sci USA 107: 16366-16371.

Ziemann U, Muellbacher W, Hallett M, Cohen LG (2001). Modulation of practice-dependent plasticity in human motor cortex. Brain 124: 1171-1181. 